\title{
Impedance Tube as a Tool for Evaluating Acoustic Noise Descriptors - The Experimental Measurement of Acoustic Parameters
}

\author{
Veronika Gumanová ${ }^{1, *}$, Laura Džuňová ${ }^{1}$, Lýdia Sobotová ${ }^{1}$ and Ružena Králiková ${ }^{1}$ \\ TUKE, Faculty of Mechanical Engineering, Department of Process and Environmental Engineering, Park Komenského 5, 04200 Košice
}

\begin{abstract}
Against the background of severe problems related to environmental noise, there has been a significant increase in the demand for its controlling. It has led to an increase in research efforts to develop the correct ways of measuring acoustic descriptors with the closely related research of soundproof and highly sound absorptive building materials. The sound insulation capacity of a barrier is indicated by two acoustic parameters - sound absorption and transmission loss. The contribution describes the principle, methods, and process of measurement of acoustics parameters using an impedance tube, explains the notion of sound absorption and transmission loss, and demonstrates this knowledge in the experimental measurement of acoustic parameters of selected materials (textiles from the automobile, polyurethane foam and absorbent cotton).
\end{abstract}

Keywords: impedance tube, acoustic descriptors, sound absorption, transmission loss.

\section{Introduction}

Compared with the measurement of the coefficient of sound absorption in an anechoic room, the impedance tube is characterized by several specific differences. An anechoic room method determines the sound absorption coefficient for diffuse sound incidence, and the technique is suitable for testing of materials with pronounced structures in the normal and lateral directions. However, an anechoic room requires expensive instrumentation, floor space, extensive testing time, and most importantly too large samples, so it is not reasonable for research work, where are available only small samples of the absorber. The impedance tube method is restricted to parametric studies at normal incidence but demands samples of the test object, which are of the identical size as the impedance tube's cross-section [1-2].

The test sample is inserted at one end of an airtight impedance tube. In the tube, waves are generated by a sound source, and the sound pressures are measured at two positions, near to the sample. The complex acoustic transfer function of the two microphone signals is assigned and applied to calculate the impedance ratio of the test sample, the normal-incidence complex reflection factor, and the normal-incidence absorption coefficient. The quantities are determined as functions of the frequency with a frequency resolution which is determined from the sampling frequency and the record length of the digital frequency analysis system used for the measurements. The usable frequency range depends on the width of the tube and the spacing between the microphone positions [3-4].

The measurements in the impedance tube may be performed by employing two types of techniques [5]:

* Corresponding author: Veronika Gumanová, E-mail: veronika.qumanova@tuke.sk 
- one-microphone technique - using one microphone successively in two positions,

- two-microphone technique - using two microphones in fixed positions.

Method 1 requires more time, generates and process signals according to particular requirements. However, it allows the selection of optimal microphone locations for any frequency by the elimination of phase mismatch between microphones. It is appropriate for the assessment of tuned resonators or precision.

Method 2 to eliminate the amplitude and phase difference characteristics between the microphones requires a pre-test or in-test correction procedure. However, it combines ease of implementation, speed and high accuracy. Method 2 is appropriate for general test purposes.

There are also three- and four-microphone method. The general theory, which focuses on the four microphone technique used for measuring normal incidence transmission loss and the absorption coefficient is presented in ASTME2611 [6]. The three-microphone impedance tube is modified a standard two-microphone impedance tube, where a third microphone is mounted on a movable hard termination. This method is conceptually identical to the four-microphone method described in the standard; however, it requires fewer transfer functions and one microphone less. Comparison of errors in the three- and four-microphone methods used in the measurement of the acoustic properties of porous materials was performed by Muehleisen and Beamer [7].

Methods of measuring the acoustic properties of materials including impedance tube were discussed by Lumnitzer et al [8]. Experimental measurement of the absorption coefficient of cork, polyethylene foam EVA and polyethylene LDPE (felt), using a two-microphone method on own constructed impedance tube was performed by Labašová and Ďuriš [9]. The measuring of sound absorption characteristics of the natural fibrous material from coconut coir, oil palm fruit bunches, and pineapple leaf using impedance tube a two-microphone method was realized by Rusli et al [10].

Table 1: Parameters and accessories of the used impedance tube BSWA TECH [11].

\begin{tabular}{|l|l|l|l|l|l|l|l|}
\hline Model & Measuring ability & $\begin{array}{l}\text { Frequency range } \\
{[\mathrm{Hz}]}\end{array}$ & $\begin{array}{l}\text { Tube diameter } \\
{[\mathrm{mm}]}\end{array}$ & $\begin{array}{l}\text { Number of microphones } \\
\text { (model MPA416) }\end{array}$ & $\begin{array}{l}\text { Hardware for } \\
\text { data collection }\end{array}$ & $\begin{array}{l}\text { Power } \\
\text { amplifier }\end{array}$ & $\begin{array}{l}\text { Software } \\
\text { SW466 }\end{array}$ \\
$\begin{array}{ll}\text { coefficient of sound absorption } \\
\text { (a) and transmission loss (TL) }\end{array}$ & $100-6300$ & 30 & 4 & $\begin{array}{l}\text { 4-Channel } \\
\text { MC3242 }\end{array}$ & PA50 & VA-LAB2 \\
\hline
\end{tabular}

\section{Experimental comparative measurement of acoustic parameters of selected materials}

2.1. Instrumentation, software and other equipment - the measuring chain

For measurements was used the impedance tube from the manufacturer BSWA TECH - model SW466. The impedance tube can accurately measure sound absorption coefficients and impedance, according to ISO10534-2. It also allows us to measure the sound transmission loss based on the Transfer Function Method (TFM). TFM can separate the reflected and incident energy from the measured transfer function, and then estimates the acoustic descriptors of the tested sample inserted in the tube. BSWA 1/4" microphones MPA416 (Fig. 2), are directly connected to optional 2-channel MC3242 data acquisition hardware. The PA50 power amplifier is used to drive the loudspeaker in the impedance tube. The BSWA VA-Lab2 software provides all measurement functions for sound absorption and transmission loss testing. The connection of the and accessories of the used impedance tube are summarized in the following Table 1 [11].

Measurement was carried out at two intervals of frequencies, $100-800 \mathrm{~Hz}$ and $400-2500 \mathrm{~Hz}$.

The system for measuring the coefficient of sound absorption (a), (for frequency bands 100 $800 \mathrm{~Hz}$ and $400-2500 \mathrm{~Hz}$ ) consists of the tube with an inner diameter of $60 \mathrm{~mm}$ and a holder of the measured sample with an inner diameter of $60 \mathrm{~mm}$.

The measuring system for the transmission loss (TL), (for frequency bands $100-800 \mathrm{~Hz}$ and 400 $2500 \mathrm{~Hz}$ ) consists of the tube with an inner diameter of $60 \mathrm{~mm}$ and a tube extension with an inner diameter of $60 \mathrm{~mm}$.

BSWA VA-Lab software disposes of the Impedance Tube Module (VA-Lab IMP) supporting measurement of sound absorption and sound insulation for BSWA SW series impedance tubes. For the capture of data and analysis, the software works with BSWA MC3242 hardware. The VA-Lab IMP can measure the absorption coefficients of material by two methods [11]:

- Method using Standing Wave Ratio (IS010534-1), measuring chain is shown in Figure 1. Parameters 


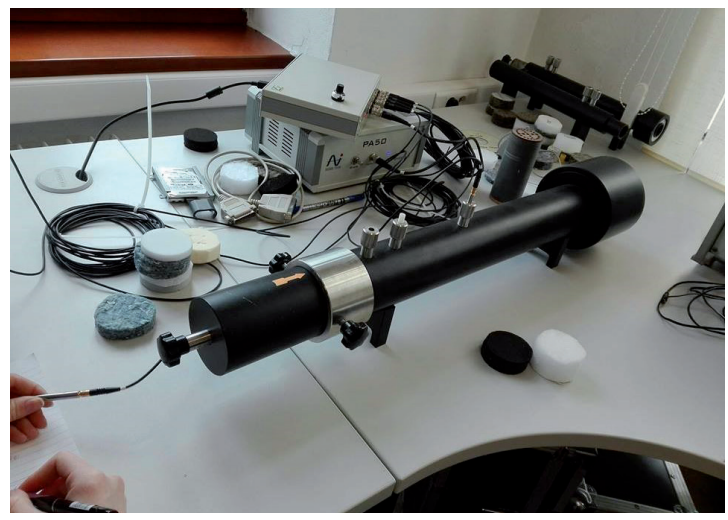

Figure 1: Connection of the measuring chain.

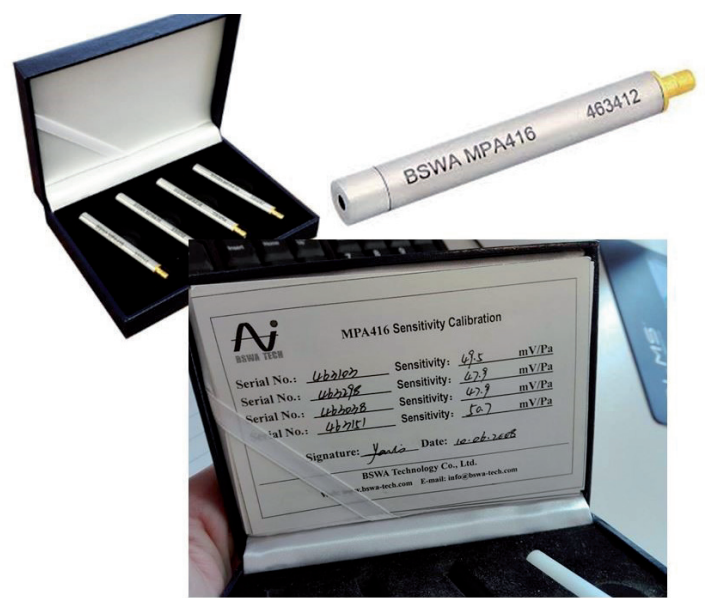

Figure 2: BSWA 1/4" microphones MPA416.

- TFM (IS010534-2).

The Standing Wave Ratio is a traditional method, which needs to generate a standing wave in the impedance tube. VA-Lab can calculate the absorption coefficients by capturing the max. and min. value of the sound pressure in the impedance tube. The other acoustic parameters, such as reflectance coefficient, impedance ratio, and admittance ratio can be calculated based on the first minimum position of the pressure.

TFM uses two fixed microphones to acquire sound pressure near the sample. VA-Lab IMP can accurately separate the incident wave and reflected wave, then calculate the absorption coefficients. An extended frequency range can be obtained from the combination of measurement results gained in different diameters of the tubes. It automatically calculates the acoustic properties of the material at a wide frequency range of interests.

The system of the signal processing consists of a

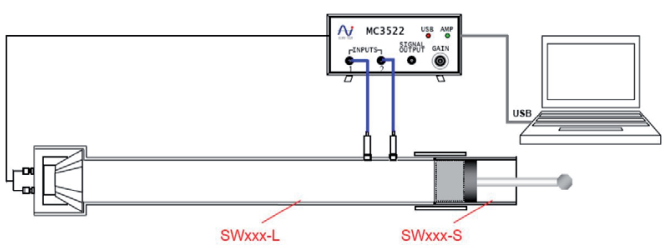

Figure 3: Measuring chain for sound absorption measurement (a) [11].

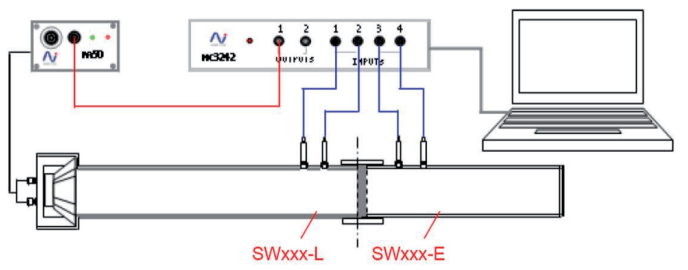

Figure 4: Measuring chain for transmission loss measurement (TL) [11].

two-channel Fast Fourier Transform (FFT) analyzing system and an amplifier. The system is demanded to measure the sound pressure at two microphone positions and to determine the transfer function $\mathrm{H}_{12}$ (see chapter 2.3.1 and eq. (9)) between them [6].

\subsection{Selection and preparation of materials}

The most abundantly used materials for acoustic testing are porous materials, due to the combination of their properties such as lightweight, low price, easy shaping and mainly excellent acoustic properties. These materials are composed of channels, cracks or cavities, which allow the sound waves entering into them, resulting in a broad frequency band for sound absorption [12].

The measurements of the selected acoustic descriptors were performed for selected three porous material - a mixture of textiles from the automobile, polyurethane foam and absorbent cotton (Fig. 5). The test specimens were prepared in the same thickness of $20 \mathrm{~mm}$, and their diameter was $30 \mathrm{~mm}$ (Fig. 6).

\subsection{Method of measurement - Transfer function method using} two microphones

2.3.1. Coefficient of sound absorption (a)

The complex sound pressure propagating in the incident and reflected direction can then be described as

$p_{I}=\hat{p}_{I} e^{\left(-j k_{0} x+\varphi_{I}\right)} e^{j \omega t}$
$p_{R}=\hat{p}_{R} e^{\left(j k_{0} x+\varphi_{R}\right)} e^{j \omega t}$

where $\hat{\boldsymbol{p}}_{\boldsymbol{I}}$ and $\hat{\boldsymbol{p}}_{\boldsymbol{R}}$ represent the magnitudes of 


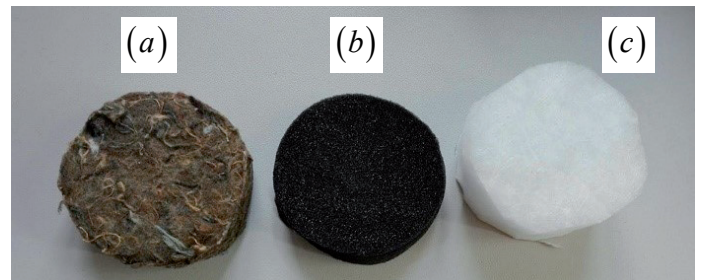

Figure 5: Samples of materials used for measurement, a) textiles from the automobile, b) polyurethane foam, c) absorbent cotton.

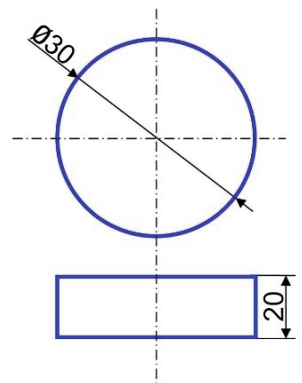

Figure 6: Dimensions of the samples.

the incident and reflecting waves, $\boldsymbol{\varphi}_{I}$ and $\boldsymbol{\varphi}_{R}$ are the phase shift of the incident and reflecting waves, $\boldsymbol{k}_{\boldsymbol{0}}$ represents the real wave number in air and $\boldsymbol{x}$ is a location in the tube from the front surface of the sample. Then are the phase and time notation subsumed in amplitude $\boldsymbol{p}_{I}$ and $\boldsymbol{p}_{R}[14]$.

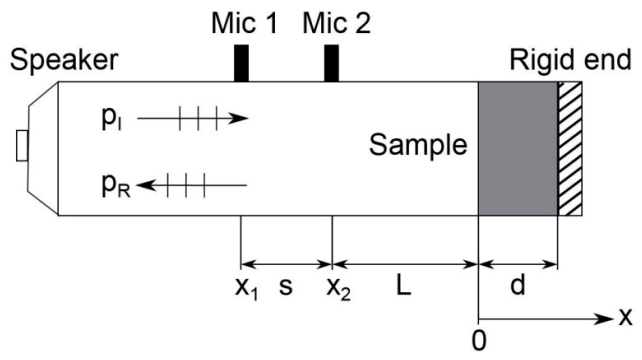

Figure 7: A scheme of an impedance tube configuration for two microphones, describing the incident and reflected wave and the positions of the microphones, speaker and sample [13].

The complex sound pressure at the location of the microphones (see Figure 7) can then be calculated as

$$
\begin{aligned}
& p_{1}=p_{I}\left(x_{1}\right)+p_{R}\left(x_{1}\right)=\hat{p}_{I} e^{-j k x_{1}}+\hat{p}_{R} e^{j k_{0} x_{1}} \\
& p_{2}=p_{I}\left(x_{2}\right)+p_{R}\left(x_{2}\right)=\hat{p}_{I} e^{j k x_{2}}+\hat{p}_{R} e^{-j k_{0} x_{2}}
\end{aligned}
$$

where $\boldsymbol{x}_{1}$ and $\boldsymbol{x}_{2}$ represent the distance from the reference point, described 0 in the mentioned figure, to microphone locations 1 and 2 respectively.
For correct internal amplitude and phase mismatch among the microphones, one can use the microphone interchange technique, also known as the microphone switching method. It means measurement is also carried out with the two microphones in a switched location, to reach the transfer function between the microphones. The switching method offers the opportunity to use non-matching and low-cost equipment because the use of the transfer function will reduce minor irregularities among the microphones.

In this paper, the general form of an exact transfer function is defined as in Equation 5. Although, each the transfer functions are in practice the estimations, $\hat{\boldsymbol{H}}$, of multiple single-sided auto-spectrum's, $\boldsymbol{A} \boldsymbol{G}$ averaged over $\mathrm{N}$ iterations (see Equation 6).

$H_{i j}=\frac{p_{j}}{p_{i}}=\frac{p_{j} p_{i}^{*}}{p_{i} p_{i}^{*}}$

$\hat{H}_{i j}=\frac{A G_{i j}}{A G_{i i}}=\frac{\frac{1}{N} \sum p_{j} p_{i}^{*}}{\frac{1}{N} \sum p_{i} p_{i}^{*}}$

And what is more, the transfer function of the incident and reflecting waves between the microphone locations are defined as

$$
\begin{aligned}
& H_{I}=\frac{p_{I}\left(x_{2}\right)}{p_{R}\left(x_{1}\right)}=\frac{\hat{p}_{I} e^{-j k_{0} x_{2}}}{\hat{p}_{I} e^{-j k_{0} x_{1}}}=e^{j k_{0} s} \\
& H_{R}=\frac{p_{R}\left(x_{2}\right)}{p_{R}\left(x_{1}\right)}=\frac{\hat{p}_{R} e^{j k_{0} x_{2}}}{\hat{p}_{R} e^{j k_{0} x_{1}}}=e^{-j k_{0} s}
\end{aligned}
$$

where $\boldsymbol{s}=\boldsymbol{x}_{2}-\boldsymbol{x}_{1}$ is the space between the microphone locations (see Figure 7). Using Equation 3 and 4 , one can obtain the transfer function $\boldsymbol{H}_{12}$ as

$$
\begin{aligned}
H_{12} & =\frac{p_{2}}{p_{1}}= \\
& =\frac{\hat{p}_{I} e^{-j k x_{0} x_{2}}+\hat{p}_{R} e^{j k_{0} x_{2}}}{\hat{p}_{I} e^{-j k_{0} x_{1}}+\hat{p}_{R} e^{j k_{0} x_{1}}}= \\
& =\frac{e^{-j\left(k_{0} x_{2}+\varphi\right)}+R e^{j\left(k_{0} x_{2}+\varphi\right)}}{e^{-j\left(k_{0} x_{1}+\varphi\right)}+R e^{j\left(k_{0} x_{1}+\varphi\right)}}
\end{aligned}
$$

where $\boldsymbol{R}$ denotes the reflection coefficient, defined as the ratio of the complex reflected and incident pressure. The equation can be in the following derived to show the reflection coefficient at microphone position $1, \boldsymbol{x}=\boldsymbol{x}_{1^{\prime}}$ by including Equation 7 and 8, 
$R_{x=x_{1}}=\frac{H_{12}-H_{I}}{H_{R}-H_{12}}=\frac{H_{12}-e^{j k_{0} s}}{e^{-j k_{0} s}-H_{12}}$

However, the reflection coefficient of interest is one of the material, which only can be reached at the location of the material surface $\boldsymbol{x}=\boldsymbol{O}$ (see Fig. 7). To compensate for the distance from microphone $\boldsymbol{I}$ at $\boldsymbol{x}_{1}$ to the surface of the sample must be added the term term to Equation 10:

$\frac{R_{x=0}}{R_{x=x_{1}}}=\frac{e^{j k_{0} x_{1}}}{e^{-j k_{0} x_{1}}}$

$R_{x=0}=R_{x=x_{1}} e^{j k_{0} 2 x_{1}}$

The reflection coefficient at the sample surface, $\boldsymbol{x}=\boldsymbol{0}$, can therefore be derived as

$R=\frac{H_{12}-H_{I}}{H_{R}-H_{12}} e^{j k_{0} 2 x_{1}}$

The general definition of the sound absorption coefficient $\alpha$ is the part of incident energy in material versus the energy impinging out. A certain number of the incident energy fraction is absorbed into the sample or rather changed to heat energy caused by the friction between the molecules of moving air and the fibrous structure inside the sample. The impedance tube is presupposed to be an acoustically closed system, this must mean the part of the incident propagating wave that is not reflected by the material is absorbed, after

$\alpha=1-|R|^{2}$

\subsubsection{Sound transmission loss (TL)}

$T L$, in general, describes the accumulated decrease in intensity of waveform energy as a wave propagates outwards from a source, or as it propagates through a certain area or a certain type of structure. In the function method, it is approximated by [14]:

$$
T L=10 \log \left(\frac{1}{\hat{0}}\right)=10 \log \left(\frac{W_{i}}{W_{t}}\right)[d B]
$$

The transmission coefficient $\tau$ represents the ratio of the incident sound power the transmitted sound power $\boldsymbol{W}_{t}$ to the transmitted sound power $W_{i}$

\subsection{Measured values}

The outputs from the measurement of the coefficient of sound absorption ( $\alpha$ ) and transmission loss (TL) for individual types of materials $20 \mathrm{~mm}$ thick are presented in Figure 8-13.
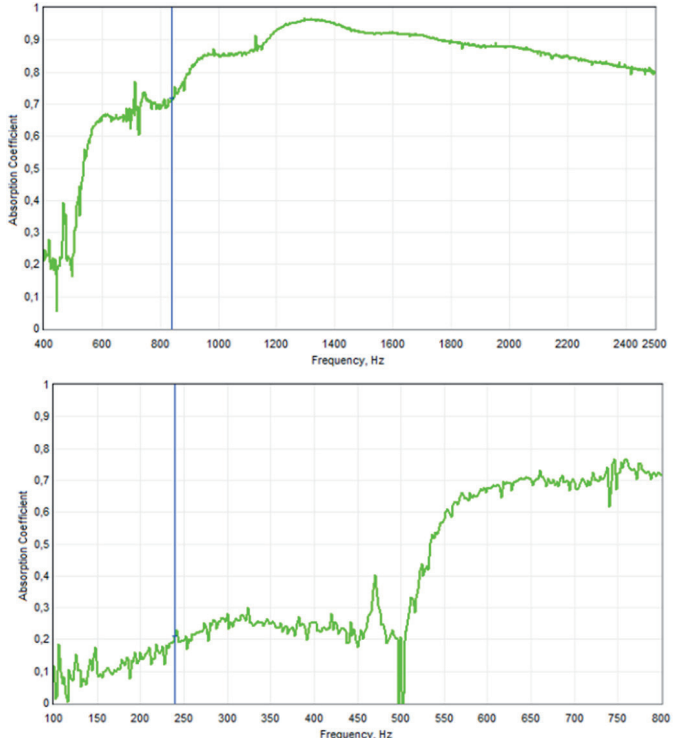

Figure 8: Textile from automobile, $a$ in the frequency range $400-2500 \mathrm{~Hz}$ (left) and 100-800 Hz (right).
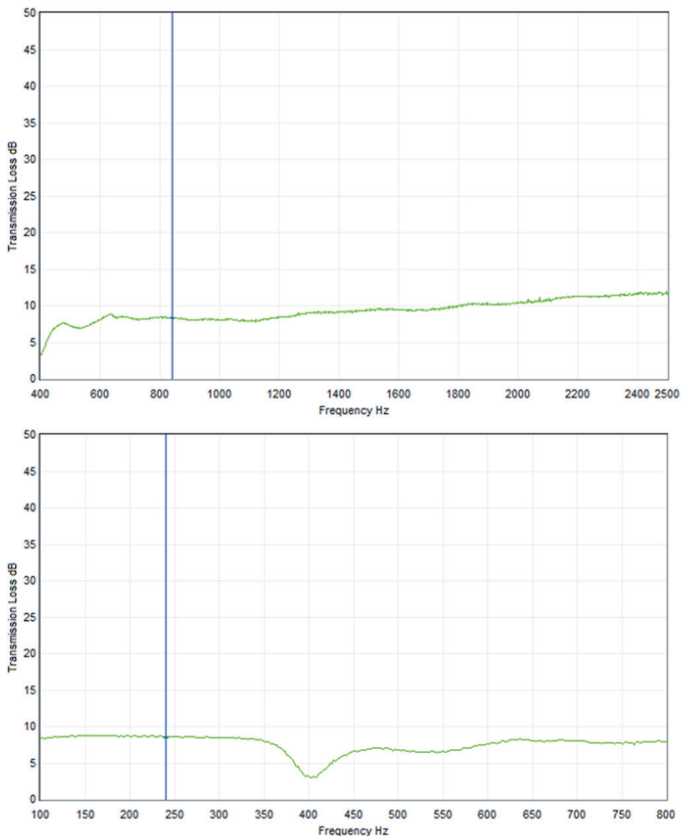

Figure 9: Textile from automobile, TL in the frequency range $400-2500 \mathrm{~Hz}$ (left) and 100-800 Hz (right). 

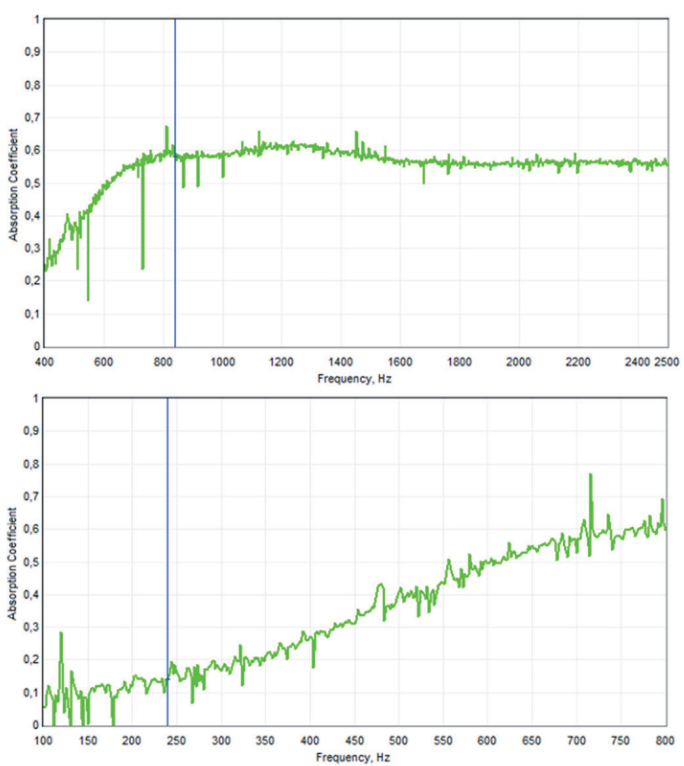

Figure 10: Polyurethane foam - $a$ in the frequency range 400$2500 \mathrm{~Hz}$ (left) and 100-800 Hz (right).
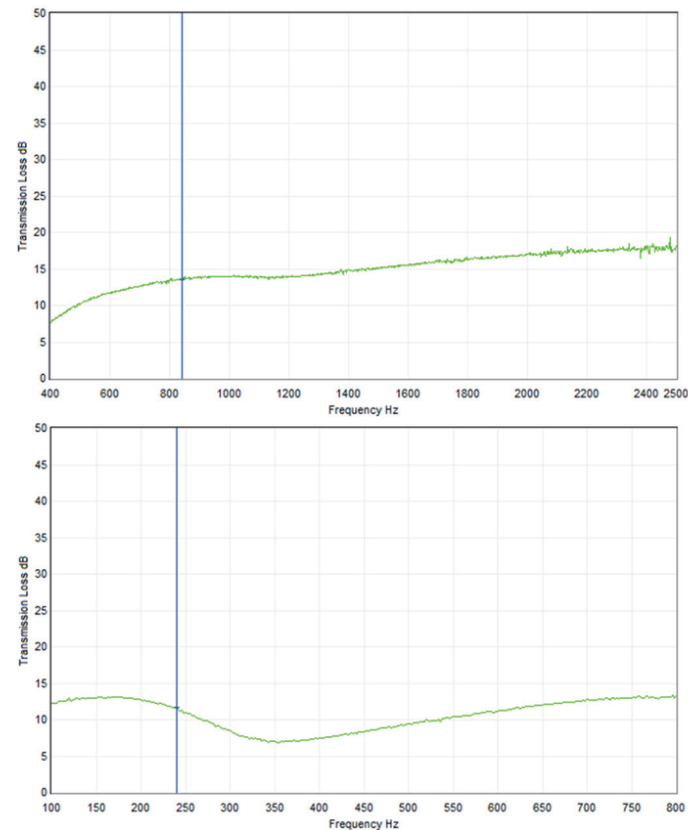

Figure 11: Polyurethane foam - TL in the frequency range 400$2500 \mathrm{~Hz}$ (left) and 100-800 Hz (right).

The blue vertical line in the graphs indicates the measured values of different materials at the same frequency and thus allows their mutual comparison. Results for all frequencies are summarized in Table $2-3$.

The significant drop of absorption coefficient in
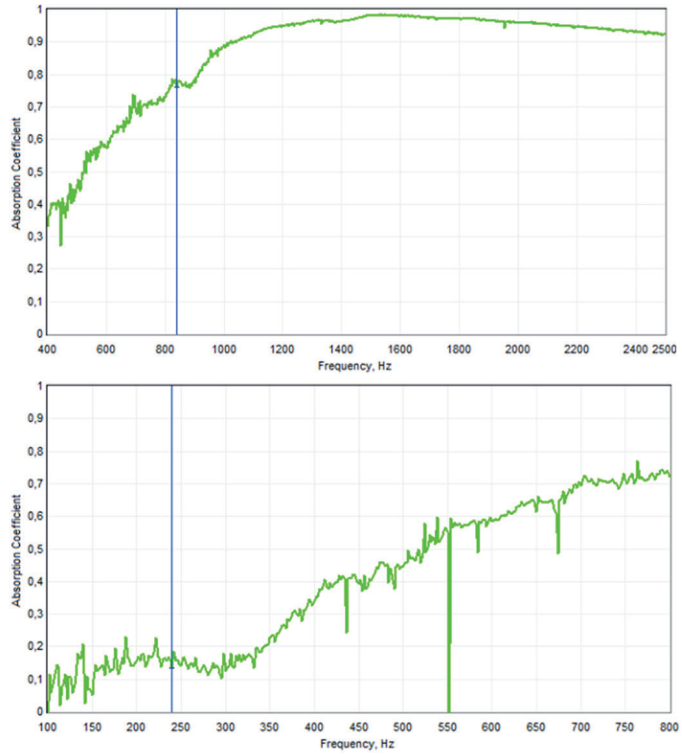

Figure 12: Absorbent cotton - $a$ in the frequency range 400$2500 \mathrm{~Hz}$ (left) and 100-800 Hz (right).
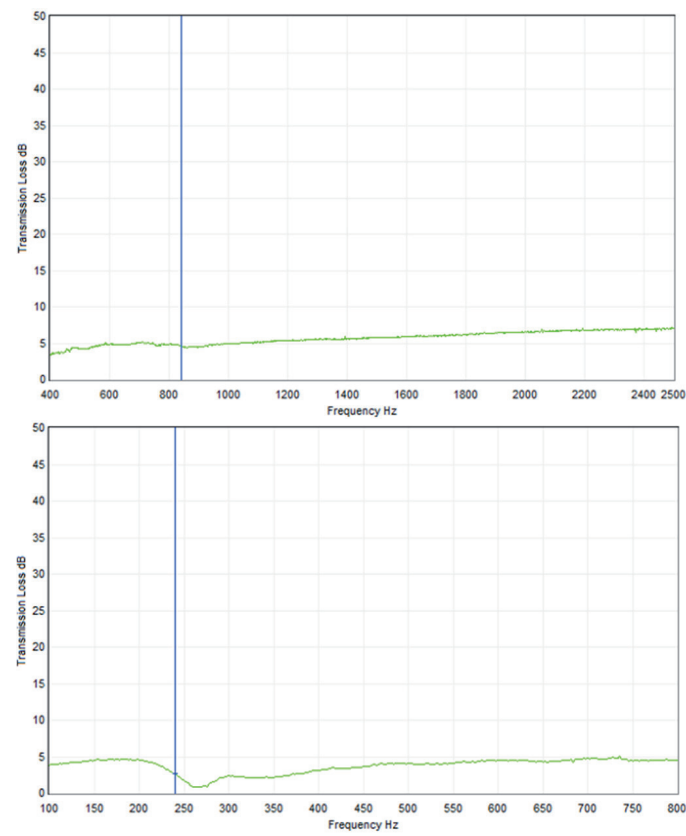

Figure 13: Absorbent cotton - TL in the frequency range 400$2500 \mathrm{~Hz}$ (left) and 100-800 Hz (right).

case of absorbent cotton at $550 \mathrm{~Hz}$ and textile from the automobile at $500 \mathrm{~Hz}$ as well as a decrease in the values of transmission loss may be caused by an error during measurement. 
Table 2: Measured values of acoustic descriptors.

\begin{tabular}{|c|c|c|c|}
\hline \multirow{3}{*}{ 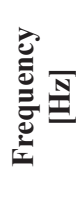 } & \multirow{2}{*}{\multicolumn{3}{|c|}{$\begin{array}{c}\text { Coefficient of sound absorption }(\alpha)[-] \\
\text { Type of material }\end{array}$}} \\
\hline & & & \\
\hline & $\begin{array}{l}\text { Mixture of } \\
\text { textile from } \\
\text { automobile }\end{array}$ & $\begin{array}{l}\text { Polyurethane } \\
\text { foam }\end{array}$ & $\begin{array}{l}\text { Absorbent } \\
\text { cotton }\end{array}$ \\
\hline 100 & 0,12 & 0,05 & 0,01 \\
\hline 125 & 0,13 & 0,08 & 0,11 \\
\hline 160 & 0,10 & 0,12 & 0,14 \\
\hline 200 & 0,16 & 0,12 & 0,15 \\
\hline 250 & 0,20 & 0,16 & 0,14 \\
\hline 315 & 0,27 & 0,20 & 0,17 \\
\hline 400 & 0,25 & 0,27 & 0,34 \\
\hline 500 & 0,21 & 0,40 & 0,45 \\
\hline 630 & 0,69 & 0,53 & 0,63 \\
\hline 800 & 0,72 & 0,60 & 0,72 \\
\hline 1000 & 0,85 & 0,59 & 0,89 \\
\hline 1250 & 0,95 & 0,61 & 0,96 \\
\hline 1600 & 0,92 & 0,57 & 0,98 \\
\hline 2000 & 0,88 & 0,55 & 0,96 \\
\hline 2500 & 0,80 & 0,55 & 0,92 \\
\hline
\end{tabular}

best results, worst results

\section{Conclusion}

The coefficient of sound absorption $(\alpha)$ is an unsized number whose values range from 0 to 1 . The measured value is closer to 1 or equal to 1, the sample of the measured absorber, and thus the absorber itself will exhibit better (higher) sound absorption. Within the coefficient of sound absorption the best and at once the worst result showed absorption cotton. The highest sound absorption was reached at a frequency of $1600 \mathrm{~Hz}(\alpha=0,98)$ and lowest in the $100 \mathrm{~Hz}$ frequency $(\alpha=0,01)$. Compare results by type of material are presented in Figure 14.

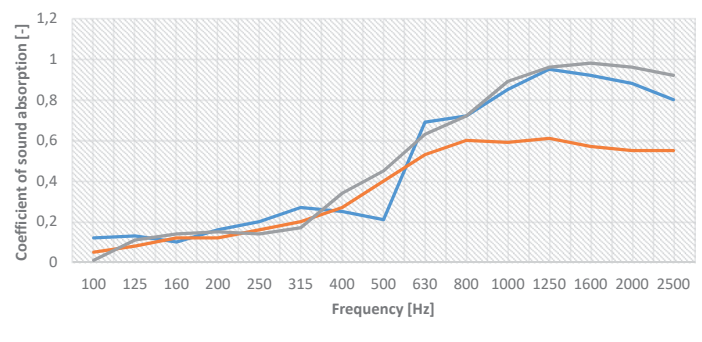

- Mixture of textile from automobile $\longrightarrow$ Polyurethane foam —Absorbent cotton

Figure 14: Compare results by type of material - Coefficient of sound absorption (a).

Transmission loss (TL) is the value that represents the damping properties of the material, which means that the higher the value, the more damping
Table 3: Measured values of transmission loss.

\begin{tabular}{|c|c|c|c|}
\hline \multirow{2}{*}{\begin{tabular}{c}
\multirow{2}{*}{} \\
\multirow{2}{*}{$\mathbf{E}$}
\end{tabular}} & \multicolumn{3}{|c|}{ Transmission loss (TL) [dB] } \\
\cline { 2 - 4 } & \multicolumn{3}{|c|}{ Type of material } \\
\cline { 2 - 4 } & $\begin{array}{c}\text { Mixture of } \\
\text { textile from } \\
\text { automobile }\end{array}$ & $\begin{array}{c}\text { Polyurethane } \\
\text { foam }\end{array}$ & $\begin{array}{c}\text { Absorbe } \\
\text { nt cotton }\end{array}$ \\
\hline $\mathbf{1 0 0}$ & 8,3 & 12,2 & 3,8 \\
\hline $\mathbf{1 2 5}$ & 8,6 & 12,7 & 4,2 \\
\hline $\mathbf{1 6 0}$ & 8,7 & 13,0 & 4,6 \\
\hline $\mathbf{2 0 0}$ & 8,6 & 12,7 & 4,6 \\
\hline $\mathbf{2 5 0}$ & 8,5 & 11,0 & 1,7 \\
\hline $\mathbf{3 1 5}$ & 8,4 & 7,7 & 2,2 \\
\hline $\mathbf{4 0 0}$ & 3,6 & 7,4 & 3,1 \\
\hline $\mathbf{5 0 0}$ & 6,7 & 9,4 & 4,1 \\
\hline $\mathbf{6 3 0}$ & 8,2 & 11,7 & 4,5 \\
\hline $\mathbf{8 0 0}$ & 7,9 & 13,2 & 4,6 \\
\hline $\mathbf{1 0 0 0}$ & 8,0 & 14,0 & 5,0 \\
\hline $\mathbf{1 2 5 0}$ & 8,6 & 14,1 & 5,4 \\
\hline $\mathbf{1 6 0 0}$ & 9,5 & 15,6 & 5,9 \\
\hline $\mathbf{2 0 0 0}$ & 10,4 & 17,0 & 6,6 \\
\hline $\mathbf{2 5 0 0}$ & 11,5 & 18,2 & 7,0 \\
\hline
\end{tabular}

- best results, worst results

the sound. TL is indicated in $\mathrm{dB}$. The most favorable result was demonstrated by polyurethane foam at a frequency of $2500 \mathrm{~Hz}(\mathrm{TL}=18,2 \mathrm{~dB}$ ), on the contrary as the earliest transmission silencer come across as absorbent cotton at a frequency rate of $250 \mathrm{~Hz}$ ( $\mathrm{TL}$ $=1,7 \mathrm{~dB}$ ). Compare results by type of material are presented in Figure 15.

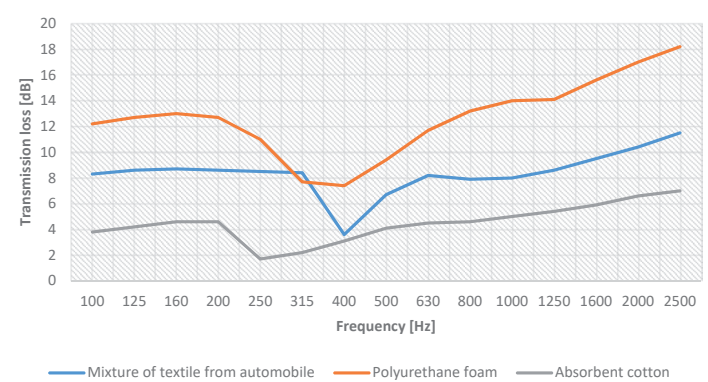

Figure 15: Compare results by type of material - Transmission loss (TL).

There are some factors, which must be considered in the interpretation of the results obtained by TFM. Transmission loss and coefficient of sound absorption is not only a property of material but is also significantly dependent on boundary conditions results from the used method, and details of the approach the sample material is installed. An incorrectly produced sample can 
cause incorrect measurement results, therefore it is important to create sample without surface irregularities and cut it exactly to the tube diameter. The sample should not be too large that it would be deformed or too small that there would be a gap. It is noted that a small pushing force applied to a sample to insert it inside the impedance tube can lead to an air space between the test sample and the rigid plunger while a big pushing force can modify the test sample (change the acoustic properties of the test sample). Pilon et al [15] investigated the effect of an air gap and found that the air gap behind the sample affects the results. The sample mounting problem and its solution is examined by Koruk [16], who recommends to utilize a fixture to insert a test sample inside an impedance tube so that there is no modification of sound absorbing properties of a test sample and air space problem.

Both, the tube's diameter and the spacing between the microphone positions determine the usable frequency range. Tubes with a smaller inner diameter allow measure at higher range of frequencies and tubes with a larger inner diameter are used for measurement at lower range of frequencies.

Measurements at different frequency ranges may cause differences in the measured results for some materials. Koruk [16] assesses the performance of the two-microphone impedance tube method as a function of frequency for different tube diameters with same material samples and presents suggestions for increasing the reliability and repeatability of impedance tube measurements. The results of Koruk's research point to similar results when measuring the same material samples using the tubes with different diameter, but there are some possible differences in the values of the absorption coefficient for samples with PU films and reflective materials.

Future research will focus on measuring the acoustic descriptors of sustainable materials (such as mycelium, hemp, coconut fibers, and others) in comparison with synthetic ones using the twomicrophone methods and different diameter of tube. We will also monitor possible differences in the measured results using a tube with a smaller and larger diameter.

\section{Acknowledgments}

This work was supported by the grant project KEGA 041TUKE-
$4 / 2018$

\section{References}

[1] Cox, T. J. - D'Antonio, P. (2016). Acoustic Absorbers and Diffusers. Theory, Design and Application. Spon Press, New York. 519 p. ISBN 0-415-29649-8.

[2] Peng, L. (2017). Sound absorption and insulation functional composites. Fan, M. - Fu, F., Advanced High Strength Natural Fibre Composites in Construction. Woodhead Publishing, United Kingdom. pp. 333 - 352. ISBN 978-0-08100411-1.

[3] ASTM E1050-98:2003. Standard test method for impedance and absorption of acoustical materials using a tube: Two microphones and a digital frequency analysis system.

[4] Cândido da Silva, G. C. (2013). Design and construction of a low cost impedance tube for sound absorption coefficient measurements. 22nd International Congress of Mechanical Engineering, Ribeirão Preto (Brazil), pp. 105 - 115. ISSN 21765480 .

[5] ISO 10534-1:1996. Acoustics - determination of sound absorption coefficient and impedance in impedances tubes - Part 1: Method using standing wave ratio.

[6] ASTM E2611-09. Standard test method for measurement of normal incidence sound transmission of acoustical materials based on the transfer matrix method.

[7] Muehleisen, R. T. - Beamer, C. W. (2002). Comparison of errors in the three- and four-microphone methods used in the measurement of the acoustic properties of porous materials. In: The Journal of the Acoustical Society of America. Vol. 3, no. 4, pp. 112 - 117. DOI: 10.1121/1.1498175.

[8] Lumnitzer, E. - Hricová, B. - Piňosová, M. (2016). Acoustical parameters of the sound absorbents. In: International journal of interdisciplinarity in theory and practice. No. 8 , pp. 16-20. ISSN 2344-2409.

[9] Labašová, E. - Duuriš, R. (2019). Measurement of the Acoustic Absorption Coefficient by Impedance Tube. In: Research Papers Faculty of Materials Science and Technology Slovak University of Technology in Trnava. Vol. 27, no. 45, pp. 94 -101. DOI: 10.2478/rput-2019-0031.

[10] Rusli, M. et al. (2019). Sound absorption characteristics of the natural fibrous material from coconut coir, oil palm fruit bunches, and pineapple leaf. Conference on Innovation in Technology and Engineering Science (IOP Conference Series: Materials Science and Engineering 602:012067). Vol. 602, no. 1. DOI: 10.1088/1757-899X/602/1/012067.

[11] BSWA Catalogue Sheet: BSWA Impedance Tube Solutions, from https://www.bswa-tech.com:8443/ web_prolnfoList. action?code=A3A0, 17.02.2020

[12] Cao, L. et al. (2018). Porous materials for sound absorption In: Composites Communication, Vol. 10, pp. 25 - 35. ISSN 
2452-2139. DOI: 10.1016/j.coco.2018.05.001.

[13] EN ISO 10534-2:1998. Acoustics. Determination of sound absorption coefficient and impedance in impedance tubes: Part 2 Transfer-function method.

[14] Vigran, T. E. (2008). Building Acoustics. Taylor and Francis, New York. 359 p. ISBN 0-203-93131-9.

[15] Pilon, D. - Panneton, R. - Sgard, F. (2004). Behavioral criterion quantifying the effects of circumferential air gaps on porous materials in the standing wave tube. In: The Journal of the Acoustical Society of America. Vol. 116, no. 1, pp. 344 - 356. DOI: 10.1121/1.1756611.

[16] Koruk, H. (2014). An assessment on the performance of impedance tube method. In: Noise Control Engineering Journal. Vol. 62, no. 4, pp. 264 - 274. DOI: 10.3397/1/376226.

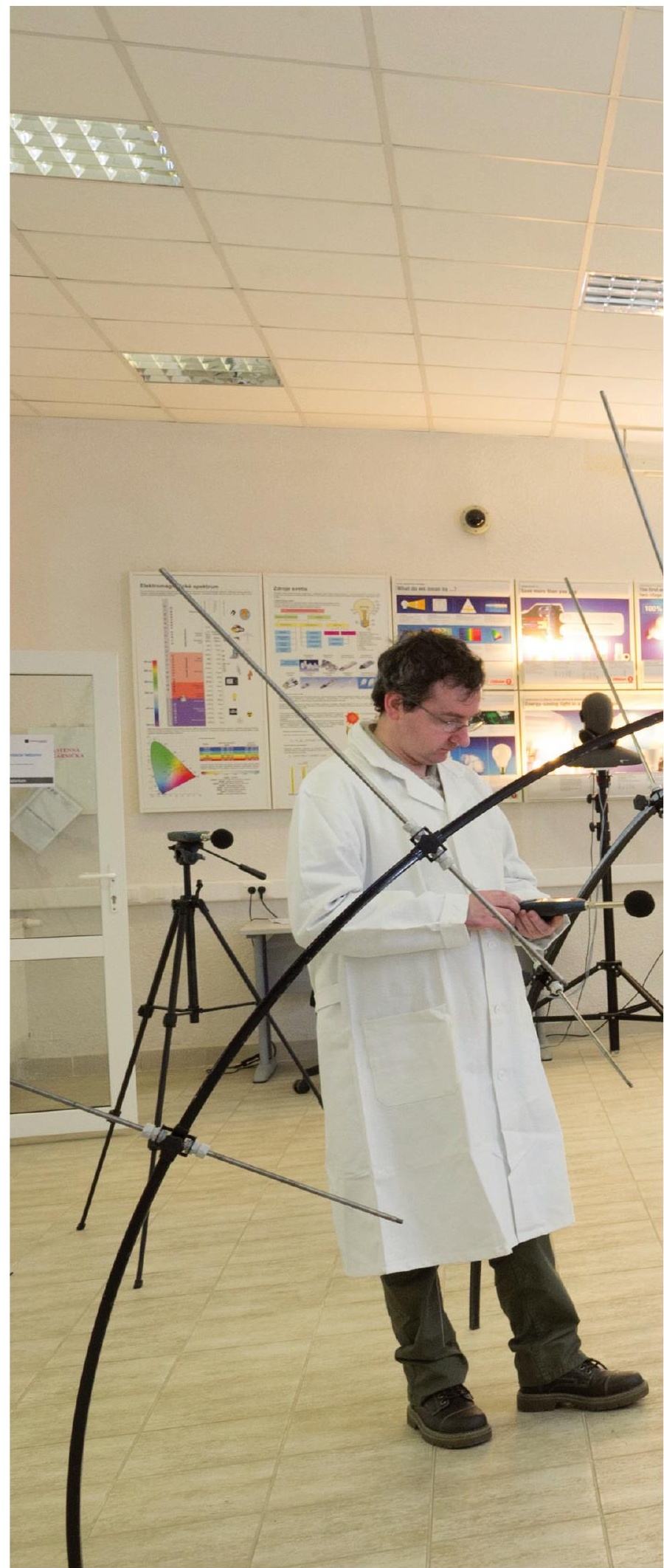

